\title{
The Kepler field of view covered with the LAMOST spectroscopic observations $\dagger$
}

\author{
Joanna Molenda-Żakowicz ${ }^{1,2}$, Peter De Cat $^{3}$, Jian-Ning Fu ${ }^{4}$, \\ An-Bing Ren $^{4}$, Antonio Frasca ${ }^{5}$ and Giovanni Catanzaro ${ }^{5}$ \\ ${ }^{1}$ Department of Physics and Astronomy, University of Wrocław, Poland, \\ email: molenda@astro.uni.wroc.pl \\ ${ }^{2}$ Department of Astronomy, New Mexico State University, Las Cruces, NM, USA, \\ ${ }^{3}$ Royal Observatory of Belgium, Brussels, Belgium, \\ ${ }^{4}$ Department of Astronomy, Beijing Normal University, Beijing, China, \\ ${ }^{5}$ INAF-Osservatorio Astrofisico di Catania, Catania, Italy.
}

\begin{abstract}
The Large Sky Area Multi-Object Fiber Spectroscopic Telescope (LAMOST) at the Xinglong observatory in China is a 4-m telescope equipped with 4,000 optical fibres. In 2010, we initiated the LAMOST-Kepler project which aimed at collecting low-resolution spectra of stars from the Kepler Input Catalog covering uniformly the Kepler field of view. The first round of the LAMOST-Kepler project has been completed in September 2014 resulting in more than 100,000 low-resolution spectra. We used those data to derive the effective temperature, the surface gravity, and the mean metallicity of our targets, as well as to detect fast rotators, and to identify emission-line stars. Our results are consistent with those reported in the literature and derived from high-resolution spectroscopy. The second round of the LAMOST-Kepler project will allow to improve the coverage of the Kepler field and to repeat observations of selected targets.
\end{abstract}

Keywords. Stars: atmospheric parameters - Surveys: spectroscopic - Space missions: Kepler Astronomical facilities: LAMOST

\section{LAMOST-Kepler Project}

The LAMOST-Kepler project, described in detail by De Cat et al. (2015), has been initiated in 2010. It's aim is to cover the field of view of the space mission Kepler (Koch et al. 2010) with low-resolution spectroscopic observations obtained for as many stars as possible. Those data will then be used to derive the stars' atmospheric parameters, i.e., the effective temperature, the surface gravity, and the metallicity, and to measure their radial and projected rotational velocity. The Large Sky Area Multi-Object Fiber Spectroscopic Telescope (LAMOST Wang et al. 1996) is the most suited instrument to perform this task. It is the largest Schmidt telescope in the world and has a circular field of view with a diameter of $5 \mathrm{deg}$ that is covered with 4000 optical fibers connected to low-resolution spectrographs.

Our list of targets has been prioritized according to the availability of the atmospheric parameters of the stars in the Kepler Input Catalog (KIC; Brown et al. 2011) and the importance of those stars for the scientific community involved in the Kepler research. From the highest to the lowest priority, our original list of targets included $\sim 120 \mathrm{MK}$ secondary standard stars, $\sim 6,500$ stars selected by the Kepler Asteroseismic Science Consortium

$\dagger$ Based on observations collected using the Large Sky Area Multi-Object Fiber Spectroscopic Telescope (LAMOST) located at the Xinglong Observatory, China. 


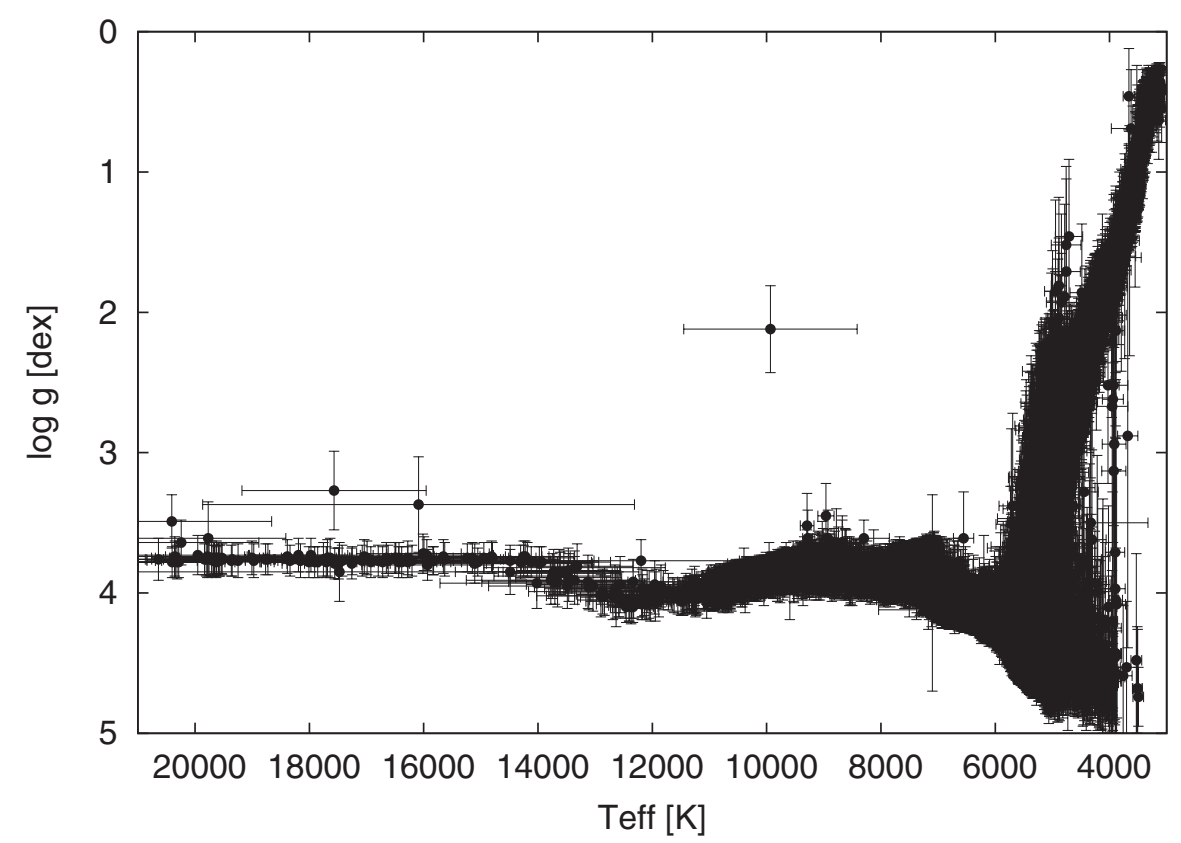

Figure 1. The $T_{\text {eff }}-\log g$ diagram obtained by the 'European team' for the stars observed with LAMOST in the framework of the LAMOST-Kepler project in the observing seasons 2011-2014.

(KASC) $\ddagger, \sim 150,000$ stars selected by the Kepler planet search group (Batalha et al. 2010), and about 1,000,000 other stars from the KIC. To allow the use of the full capacity of the LAMOST, we consulted the USNO-B catalog (Monet et al. 2003) to select additional targets with $V<20$ to fill as many fibers as possible. Our list of targets included also stars from the open clusters NGC 6791, NGC 6811, NGC 6819, and NGC 6866. We requested observations for 14 LAMOST to fully cover the Kepler field of view, as shown in Fig. 2 of De Cat et al. (2015).

The first round of the LAMOST-Kepler project was completed in 2014. Between 2011 and 2014, we measured 38 LAMOST plates during 27 observing nights and collected 101,086 low-resolution spectra $(R=1000$ or 2000 in 2011 and $R=1800$ from 2012 onwards) for 80,447 different stars; 17,114 stars were observed more than one time.

\section{Methods of Analysis}

The spectra collected in the framework of the LAMOST-Kepler project are now being analyzed by three teams with independent methods (see De Cat et al. 2015 for the details.)

The 'Asian team' (Ren et al. in preparation) and the 'European team' (Frasca et al. in preparation) both obtain the atmospheric parameters of the stars, their radial velocity, and the spectral classification. Additionally, the 'European team' measures the projected rotational velocity of stars with $v \sin i \geqslant 150 \mathrm{~km} \mathrm{~s}^{-1}$. In Fig. 1, we show a preliminary Kiel diagram obtained for the stars observed with LAMOST in the seasons 2011-2014

$\ddagger$ Relevant information on KASC can be found on the Kepler Asteroseismic Science Operations Center (KASOC) webpage http://kasoc.phys.au.dk/ maintained by Rasmus Handberg from the University of Birmingham in the United Kingdom. 
and analyzed by the 'European team' by using the code ROTFIT (Frasca et al. 2003, 2006).

The 'Asian' team performs also the statistical analysis of the derived parameters.

The 'American team' provides the MK classification by means of the code MKCLASS described by Gray \& Corbally (2014). The code is able to detects stars with peculiar features in the spectra, i.e., e.g., barium stars, Ap and Am stars, $\lambda$ Bootis stars, etc (Gray \& Corbally, in preparation).

\section{Future of the Project}

At the beginning of the LAMOST-Kepler project, the LAMOST instrument experienced some pointing problems and malfunctions of some of the spectrographs and fibers. As a result, a significant fraction of the spectra obtained by that time could not be reduced by the reduction pipeline (see Table 3 of De Cat et al. 2015.) Moreover, a good LAMOST spectrum is only available for $17 \%$ of the stars that have been observed by Kepler so far. We therefore requested a second round of the LAMOST observations during which we will focus on those targets observed by Kepler for which no LAMOST spectrum with a signal-to-noise ratio above 20 in the $r$ band is available yet. The second priority will be given to those stars already observed by Kepler for which a good quality LAMOST spectrum was obtained in the first round of the LAMOST-Kepler project. That will allow us to detect possible radial velocity variations of our targets.

The second round of the LAMOST-Kepler has already started. On 29 and 30 May 2015, two LAMOST-Kepler fields were observed (LK14 and LK11, as given in Table 1 of De Cat et al. 2015.) These observations will continue during the next observing seasons. Also the possibility of observing fields of view of the K2 mission (Howell et al. 2014) is being discussed.

\section{Acknowledgements}

The research leading to these results has received funding from the European Community's Seventh Framework Programme (FP7/2007-2013) under grant agreement no. 269194. JM-Ż acknowledges the NCN grant number 2014/13/B/ST9/00902.

\section{References}

Batalha, N. M., et al. 2010, ApJ Letters, 713, L109

Brown, T. M., Latham, D. W., Everett, M. E., \& Esquerdo, G. A. 2011, AJ, 142, 112

De Cat, P., et al. 2015, arXiv: 150806391, accepted for publication in ApJS

Frasca, A. et al. 2003, A\&BA, 405, 149

Frasca, A. et al. 2006, $A \mathscr{E} A, 454,301$

Gray, R. O. \& Corbally, C. J. 2014, $A J, 147,80$

Howell, S. B. et al. 2014, PASP, 126, 398

Koch, D. G. et al. 2010, ApJ Letters, 713, L79

Monet, D. G. et al. 2003, AJ, 125, 984

Wang, S.-G., Su, D.-Q., Chu, Y.-Q., Cui, X., \& Wang, Y.-N. 1996, Applied Optics, 35, 5155 